\title{
Vaginoplasty in mayer Rokitansky-Kuster-Hauser syndrome using amnion: a case series
}

\author{
Rachna Chaudhary*, Vandana Dhama, Shakun Singh, Renu Azad
}

Department of Obstetrics and Gynecology, LLRM Medical College, Meerut, Uttar Pradesh, India

Received: 23 August 2016

Revised: 24 September 2016

Accepted: 24 September 2016

\section{*Correspondence:}

Dr. Rachna Chaudhary,

E-mail: drrachnachaudhary@ gmail.com

Copyright: (c) the author(s), publisher and licensee Medip Academy. This is an open-access article distributed under the terms of the Creative Commons Attribution Non-Commercial License, which permits unrestricted non-commercial use, distribution, and reproduction in any medium, provided the original work is properly cited.

\section{ABSTRACT}

Background: Congenital absence of both uterus and vagina is termed Mullerian aplasia, Mullerian agenesis, or Mayer-Rokitansky-Kuster-Hauser syndrome. In classical mullerian agenesis patients have a shallow vaginal pouch, only measuring 1 to 2 inches deep. In addition the uterus, cervix, and upper part of the vagina are absent. One treatment goal for most of these women is creation of an artificial vagina either conservatively or surgically. McIndoe procedure is the most commonly employed surgical approach for creation of neovagina. The neovagina thus created is lined with skin graft, amniotic membrane, cutaneous and myocutaneous flaps, buccal mucosa, and absorbable adhesion barrier.

Methods: This study was conducted on 8 patients presenting with MRKH syndrome over a period of 3 years from 2012-2015. All the patients were admitted in SVBP hospital associated to LLRM Medical College, Meerut, (UP). McIndoe procedure was the surgical treatment common to all patients, where the neovagina created was lined by amniotic membrane. The surgery was performed by two consultants using the standardized surgical technique.

Results: In our study, all patients were followed for preferably 6 months (1,4,8,12 weeks and if possible 6 months) after surgery for following observations-vaginal caliber, presence of any stricture, vaginal sloughing, failure of graft uptake any urogenital complaints. Except for complication in one patient, all the rest had excellent results.

Conclusions: Vaginoplasty using amnion as a graft is a safe, effective treatment in patients of MRKH Syndrome. When done by experienced gynecologist, major post operative complications seem to be very few.

Keywords: Amnion graft, MRKH Syndrome, Vaginoplasty

\section{INTRODUCTION}

Vaginoplasty is a major surgical procedure, where an artificial vaginal cavity is created either in cases of congenital absence of vagina due to vaginal agenesis or in the case of male to female sexual or gender reassignment surgery (SRS or GRS) where a vaginal cavity did not exist.

Mullerian dysgenesis occurs in approximately 1in 5000 live born females. Mayer-Rokitansky-Kuster-Hauser (MRKH) syndrome is a disorder that occurs in females and mainly affects the reproductive system. This condition causes the vagina and uterus to be underdeveloped or absent. Affected women usually do not have menstrual periods due to the absent uterus and often this is how these patients present, as a case of primary amenorrhea. Women with MRKH Syndrome have a female chromosome pattern (46 XX) and normally functioning ovaries. They also have normal female external genitalia and normal breast and pubic hair development. Although women with this condition are usually unable to carry a pregnancy, they may be able to have children through assisted reproduction. Type 1 MRKH Syndrome is characterized by an isolated absence of the proximal two thirds of the vagina, whereas type 2 
is marked by other malformations, including vertebral, cardiac, urologic (upper tract), and otologic anomalies. The goal of the treatment is to provide the patient with an unscarred vagina that allows sexual functioning. ${ }^{1}$

Despite clear understanding of anomaly of Mullerian agenesis in a particular patient, a correct form of defect may not be established until the time of surgical correction. A sound knowledge of surgical anatomy of this region is of paramount importance to have successful outcome of this procedure. A thorough counselling of the patient along with parents regarding her modified marital prospects concerning sexuality, getting married to a person not keen to have children out of the married relation, social and financial self-sufficiency, etc. are of utmost importance.

\section{Surgical anatomy}

The reproductive system in the female consists of external genitalia, gonads and an internal duct system.

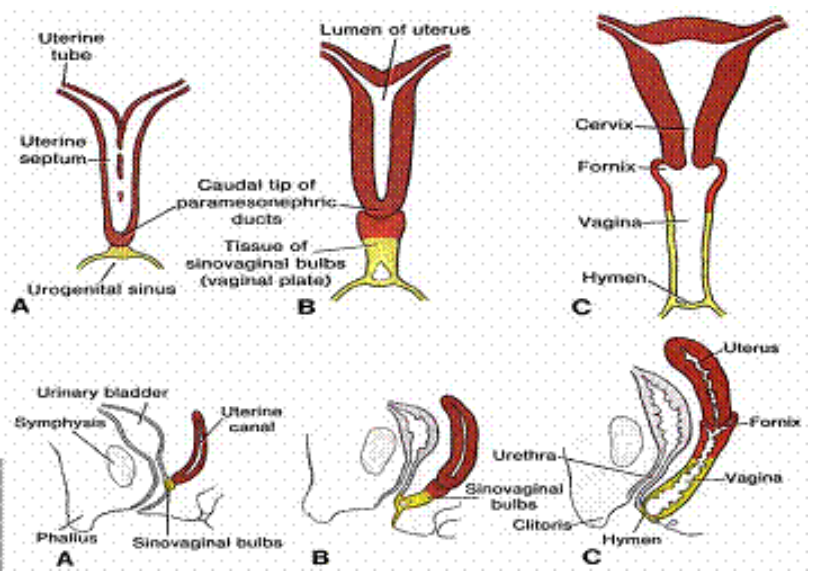

Figure 1: Development of female reproductive system.

Embryologically, these components originate from different primordia and in close association with the urinary system and hindgut (Figure 1). The Mullerian duct system is stimulated to develop preferably over the Wolffian duct system, which regresses in early female fetal life. The Mullerian ducts persist and attain complete development to form the Fallopian tubes, the uterine corpus and cervix and a portion of the vagina. Vagina is formed partly from Mullerian ducts and partly from urogenital sinus (Figure 1).

A solid vaginal cord results from proliferation of cells at the caudal tip of the fused Mullerian ducts. The cord gradually elongates to meet bilateral sinovaginal bulb developing from the urogenital sinus below. Subsequent canalization of vagina and epithelialization with cells mostly derived from urogenital sinus leads to complete formation of vagina. Hymen is the embryological septum between the sinovaginal bulbs above and urogenital sinus below which is not derived from the Mullerian ducts. Failure in the development of Mullerian duct at any time between their origins from coelomic epithelium to fusion with urogenital sinus is the underlying basis of different forms of vaginal agenesis. Failure of development of a Mullerian duct may also be associated with failure of development of ureteric bud from the caudal end of the Wolffian duct leading to congenital absence of kidney on the side ipsilateral to the agenesis of a mullerian duct. A significant percentage of these female patients with congenital absence of the kidney may be found to have associated genital anomalies., ${ }^{2,10}$

\section{Indications of surgery}

- $\quad$ Congenital absence of uterus and vagina, commonly known as Mayer-Rokitansky-Kuster-Hauser syndrome is the most common indication of vaginoplasty.

- However, an incomplete form with transverse vaginal septum or partially atretic vagina with intact uterus also requires vaginoplasty.

- In Testicular feminization syndrome or Androgen insensitivity syndrome, the vagina may be incompletely developed because of the existing male gonadal suppression of the development of the Mullerian ducts. These genetically male patients with female phenotype, without a completely formed vagina should be surgically corrected in order to have a satisfactory sexual function in their female gender role.

- Similarly, an ineffective suppression of mullerian ducts may lead to ambiguous genitalia associated with small rudimentary uterus or a partially developed vagina requiring vaginoplasty in congenital adrenal hyperplasia.

- In the case of male to female sexual or gender reassignment surgery (SRS or GRS) where a vaginal cavity did not exist.

\section{Preoperative evaluation}

The timing of start of treatment must be decided at around 17-20 years of age when the patient is emotionally mature and intellectually reliable enough to manage without difficulty the vaginal mould that will be used to maintain the neovaginal space. Alternatively, vaginoplasty may be planned a few months prior to marriage in order to initiate regular sexual activity for maintenance of vagina patency.

\section{Investigations}

- Abdominal Ultrasonography helps in revealing an absent uterus and may differentiate between hematocolpos, hematometra, endometrial and other ovarian cysts and pelvic kidney. Scan for other renal anomalies should also be done.

- Intravenous pyelogram to delineate the renal anomalies.

- $\quad$ MRI may be diagnostic for both uterine, vaginal and renal findings. 


\section{Different approaches to construct vagina}

There is no consensus regarding the correct approach to the problem of vaginal agenesis. Both surgical and nonsurgical methods of correction have their benefits and limitations.

\section{Nonsurgical methods}

\section{Frank technique or perineal dilatation}

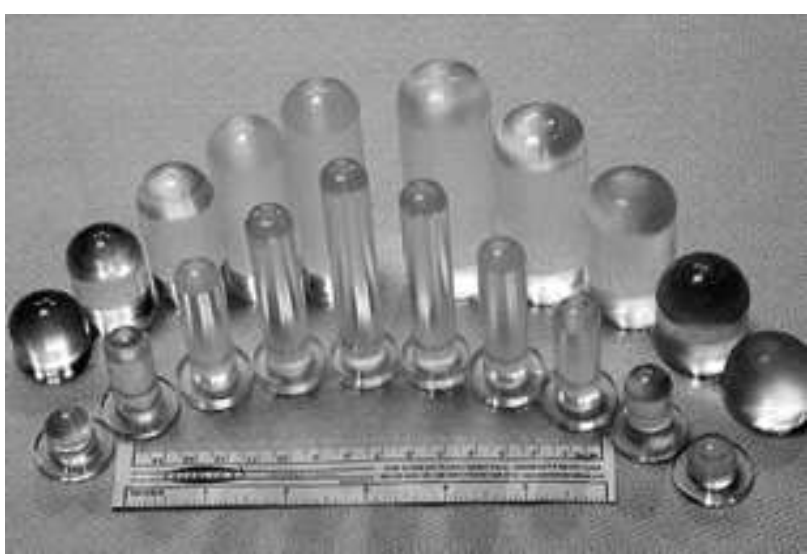

Figure 2: Perineal dilators.

The basis of this approach is the graduated dilatation of vaginal dimple at the introitus. Dilators (Figure 2) are placed against the fourchette and firm pressure is kept up for 15 minutes twice a day or more often or using a bicycle seat stool. The dilatation will go further slowly till a full length vagina can be achieved. The technique is self-administered and requires time and patient motivation. Compliance may be poor in patients with a vaginal dimple or no vagina, because these patients may experience discomfort and abandon the dilator. The patient's enthusiasm must be reinforced by the attendant supervising her case. ${ }^{3,8}$

\section{Surgical methods}

The surgical techniques include:

1. Mcindoe procedure: This procedure was first described by Abbe, further modified by McIndoe and Banester, and a number of researchers. In this operation, a space is created between rectum and bladder, largely by blunt dissection through a $0.5 \mathrm{~cm}$ incision made across the region of fourchette. Blunt finger dissection is then carried out to achieve optimum vaginal length of $10-12 \mathrm{~cm}$. The space is then lined by split skin graft taken from the buttock, thigh, or suprapubic region/or amnion graft (Figure 3 ). The graft is then sewn over a suitable mould with deep surface of the graft facing outward so that it will be in contact with the newly created vaginal wall. The moulds may be rigid over of dental stent or malleable made from rubber foam packed into rubber condoms. McIndoe originally left the mold in place for 3 months to avoid contraction of new vagina. ${ }^{8-10}$

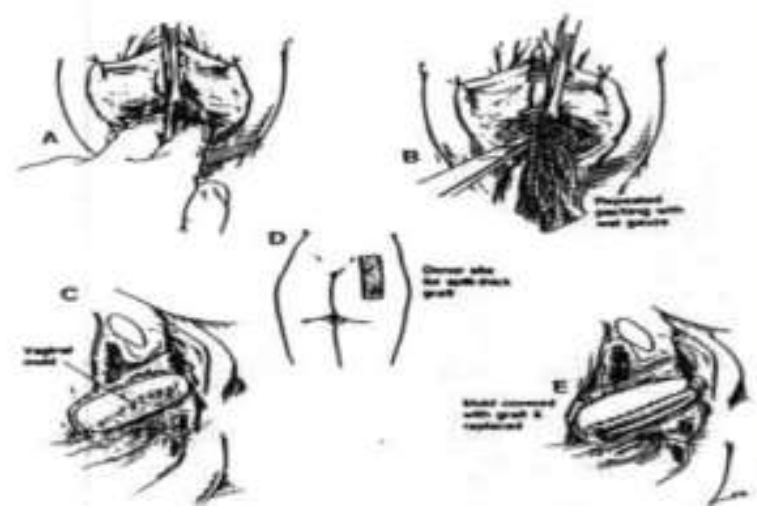

Figure 3: McIndoe procedure.

2. Colon vaginoplasty: This procedure uses a segment of rectosigmoid colon as a covering structure in place of split thickness graft after creating a vaginal space in a similar manner as in McIndoe operation. ${ }^{6}$

3. William vulvovaginoplasty: In this operation, a horseshoe-shaped incision is made in the vulva lateral to the midline and passing superior to the urethral orifice for similar distance after insertion of an indwelling catheter in the bladder. The base of the incision thus passes across the region of fourchette in a gentle curve. Edges are freed by gentler undercutting and the internal edges on each side are stitched together in the midline with knots inside the vaginal lumen. Finally, the internal skin margins are approximated with interrupted sutures (Figure 3). It should be possible to insert two fingers into the pouch to a depth of $3 \mathrm{~cm}$.

An advantage of this technique is its technical simplicity where no mould is needed, and hence,the new vagina has no tendency to contract even though it is neither dilated nor used for natural coitus for some time after the operation. ${ }^{8-10}$

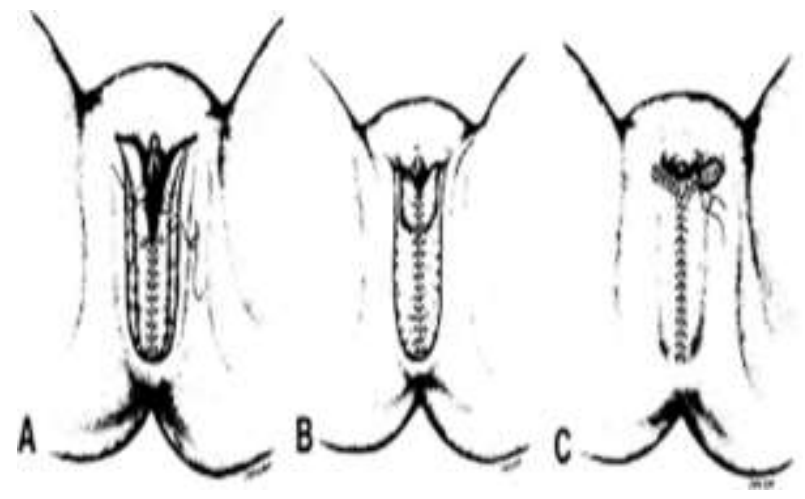

Figure 4: William vulvovaginoplasty.

4. Laparoscopic neovaginoplasty (Vecchietti procedure): Vecchietti operation originally described 
in 1965 can be considered as surgical version of Frank's method of vaginal graduated dilatation. Constant pressure is exerted on vesicorectal space from above to an acrylic olive, which is pulled upward, causing a deep invagination in this space in 7-8 days. The constructed new vagina must be maintained by daily application of artificial dilators. This technique is now performed laparoscopically.

\section{Postoperative care}

All the techniques of vaginoplasty need a meticulous postoperative care and follow up for neovagina to maintain its desirable shape and size. After 1 week of surgery, labial sutures are removed and the cavity is inspected for healing and progress of epithelialization. The mould is washed and patient is demonstrated a reinsertion so as to continue self-dilatation of the neovaginal cavity. The patient is instructed to continue dilatation of neovagina four to six times a day to a total period of 45 minutes per day. Initially, this dilatation appears cumbersome and painful, however, with time it becomes less frequently required. The neovagina has a strong tendency to shrink at any stage of life in absence of regular sexual intercourse or alternative artificial dilatation of the neovagina. Hence, a neovagina requires a lifelong maintenance in order to retain its benefits.

\section{Complications}

Vaginal stenosis is by far the most common and the most significant complication of vaginoplasty. Indeed a long period of not dilating can result in shrinkage of neovagina in terms of both diameter and length. With careful perseverance and a little discomfort this loss can usually be recovered. Failure of primary surgery usually results in infection and formation of fibrotic vaginal bands. Repeat surgery in such patients carries the greatest risk of fistula formation because the dissection through tough fibrous tissue is quite difficult as compared to loose soft tissues at the first operation. Fistula formation is the most serious complication, if it occurs during operation, the original procedure should be abandoned and treatment to close the fistula should be instituted first. Pulmonary embolism from deep vein thrombosis is another complication that can be avoided with intermittent leg compression and anticoagulant therapy. It is not unusual to notice some spraying of urine during micturition; this usually resolves itself as genital swelling subsides. Patient should be reassured regarding natural consequences of new vaginal geography and tendency to get used to with time. ${ }^{4,5,9}$

\section{METHODS}

This study was conducted on eight patients of vaginal agenesis admitted to gynaecology ward of SVBP Hospital associated to LLRM Medical College, Meerut; from 2012-2015. Allpatients presented to gynae OPD as a case of primary amenorrhoea (absence of menstruation by age 16 in the presence of secondary sexual characteristics). The patients were in the age group 18-20 yrs.

As per the protocol observed in examining a pt. of primary amenorrhoea, examination was carried out methodically. All the pt. were of average stature and had well developed secondary sexual characteristics (breast, axillary and pubic hairs). Local examination revealed normal external genitalia but with blind vagina. A detailed pelvic and renal ultrasound was carried out which revealed absent uterus in all cases but with presence of ovaries. No patient had any renal anomaly or any related urinary complaint.

Vaginoplasty is been done in our hospital for other indications as well, like androgen insensitivity syndrome, secondary vaginal atresia, vaginal septum, but for want of uniformity only cases of MRKH Syndrome were selected for vaginoplasty using amnion. As, no patient had any renal anomaly, IVP was not done. Other relevant preop investigations like hemogram, blood group, serum urea, creatinine, blood sugar, sr. bilirubin, urine routine and microscopy examination, ECG and chest X-ray were carried out.

A detailed counselling of patients and parents were carried out. The whole procedure, the possible complications and the need for a stringent follow up was carefully explained. As we have used amnion to line the neo-vaginal space, verbal and written consent to use a foreign tissue from another person was taken. All queries regarding their fears of transfer of HIV and Hepatitis were sorted out. In spite of being fit for surgery, the patients graciously waited as their surgery was to be kept on the day of elective caesarean section, from which fresh amnion was used. Bowel preparation was done and injectab le antibiotic (ceftriaxone+sulbactum) were started on the night prior to surgery.

\section{Preparation of amniotic membrane}

Amniotic membrane was obtained under sterile conditions after elective caesarean delivery from a seronegative donor or it may be stored in normal saline at 4 degree $\mathrm{C}$ for $48-72$ hours. The amniotic membrane with meconium staining, suspected chorio-amnionitis or premature rupture should not be used for grafting. The sera from all amnion donors were tested for hepatitis B, hepatitis C, HIV, Syphilis. The placenta is washed free of blood clots with balanced saline solution containing antibiotics.

The inner amniotic membrane is separated from the rest of the chorion by blunt dissection (through the potential spaces between these two tissues). The amnion can be peeled off from the fetal surface of the placenta except at the insertion of the umbilical cord. The outer surface of amnion is opposed to the inner aspect of the chorion from which it can be peeled off (Figure 5). The use of amniotic 
membrane over the mould eliminates the need for skin grafting. ${ }^{7-10}$

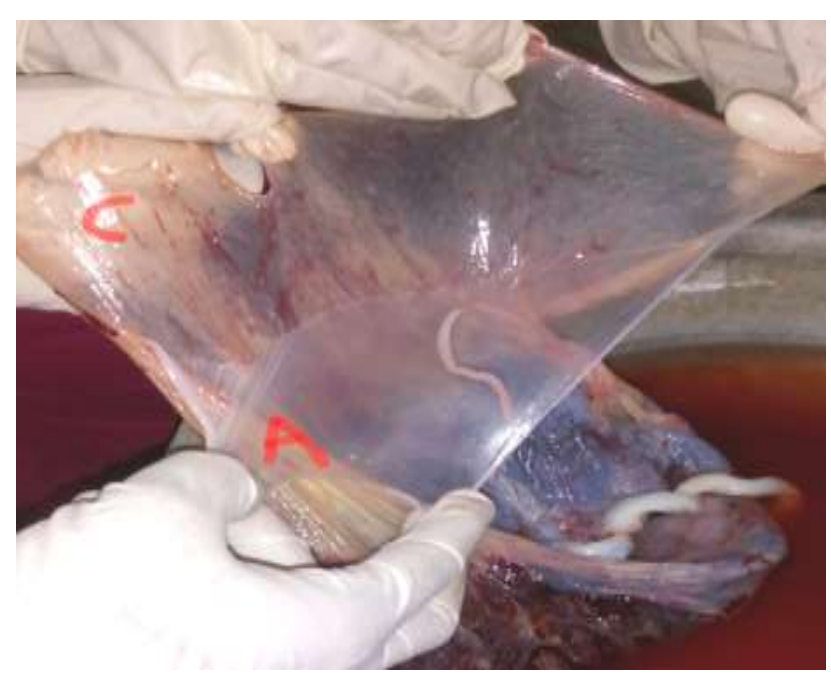

Figure 5: Preparation of amniotic membrane.

\section{Surgical technique}

The surgical technique used was McIndoe vaginoplasty with Sheares modification.

Two different gynaecologists (experience more than ten years) performed the operations.

After regional aneasthesia, patient was placed in lithotomy position with legs in stirrup was made. Parts cleaned draped. Bladder was catheterized with foley's catheter. A small amount of methylene blue was instilled through catheter into bladder so that inadvertent injury because of faulty dissection or in the wrong plane could be diagnosed at its earliest. We used Hegar dilators to stretch up the region of paramesonephric duct on each side of a midline septum between them, to make two openings admitting little finger each of approx. $4-5 \mathrm{~cm}$ length (Figure 4).

The midline septum was then divided by scissors to make a single cavity accommodating two fingers breadth wise with ease. Further dissection was done cephalad with extreme gentleness and caution as dissection beyond this point increases the chances of injuring bladder anteriorly and rectum posteriorly. A gloved finger of assistant in rectum will act as guide to prevent excessive dissection and burrowing posteriorly and avert perforation. Similarly, the Foley catheter may serve as an orientation tool anteriorly. When in doubt about whether in the right plane or not an empty syringe with needle can be used to aspirate through anterior vaginal wall. If blue coloured fluid is aspirated it means that the plane is wrong and we are more anteriorly towards bladder.

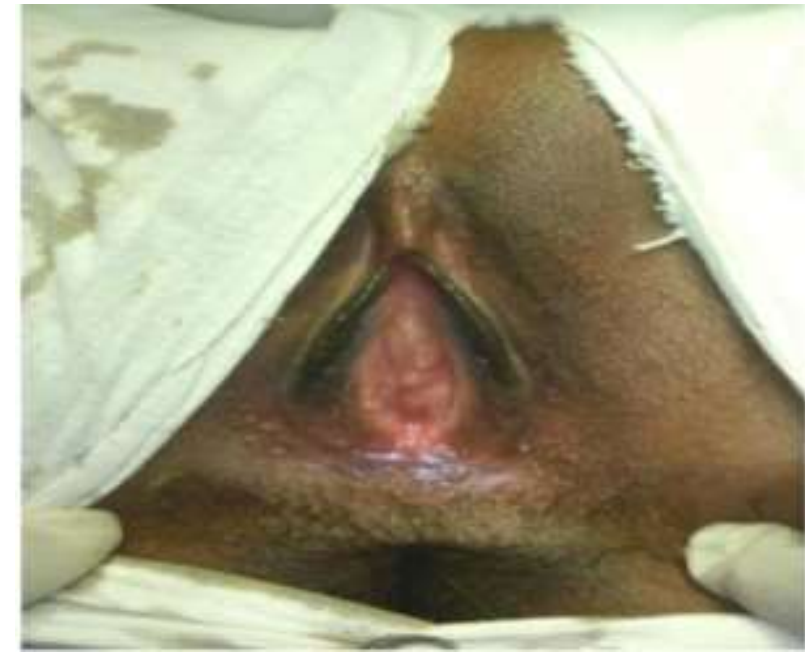

Figure 6: Preop blind vagina.

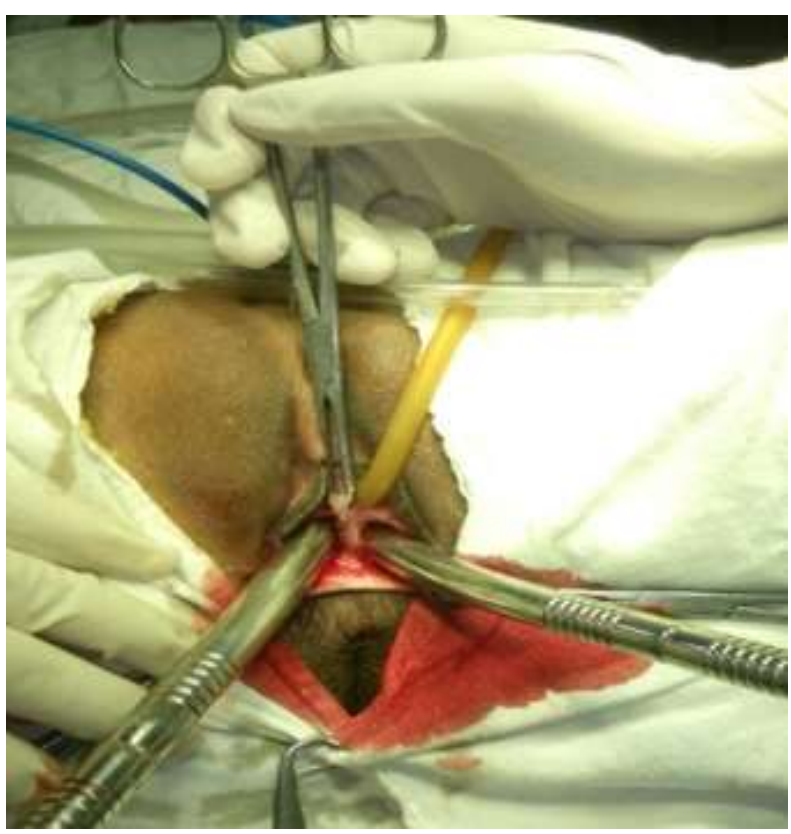

Figure 7: Sheares modification of using dilators.

To expand the space, retractors can be placed along the lateral walls of the forming canals and stretched outward. Moreover, incising the medial fibres of the puborectalis muscles can add further width. These muscles are cut along the lateral aspect of each canal and at a level midway along the antero-posterior length of the canals.

Cephalad, the canal is extended to within $2 \mathrm{~cm}$ of the culde-sac of Douglas. This leaves a layer of connective tissue affixed to the peritoneum. The skin graft will attach more effectively to this connective tissue than to a smooth peritoneal surface. The final single canal measures approximately 10 to $12 \mathrm{~cm}$ deep and three fingerbreadths wide. 


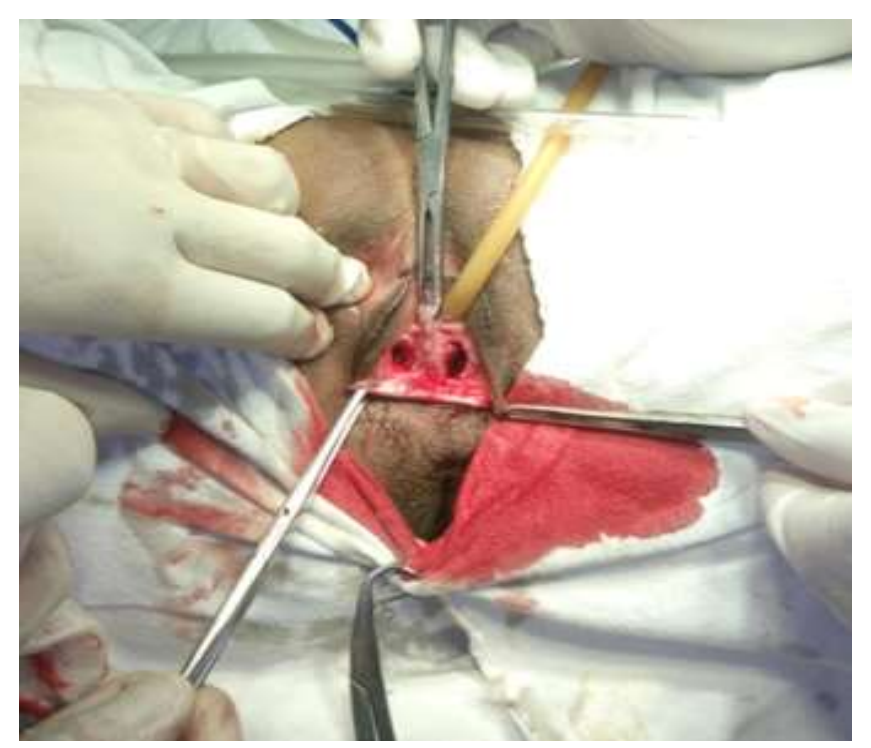

Figure 8: Creating neovagina.

As collections of blood can separate the skin graft from the canal beds, hemostasis is required prior to mould insertion. So, by the time an appropriate mould is prepared the neovaginal cavity is packed with saline soaked vaginal pack to stop oozing. Adrenaline should not be used as it causes capillary vasoconstriction and the subsequent graft uptake would be hindered. ${ }^{1,7-10}$

\section{Vaginal mould preparation}

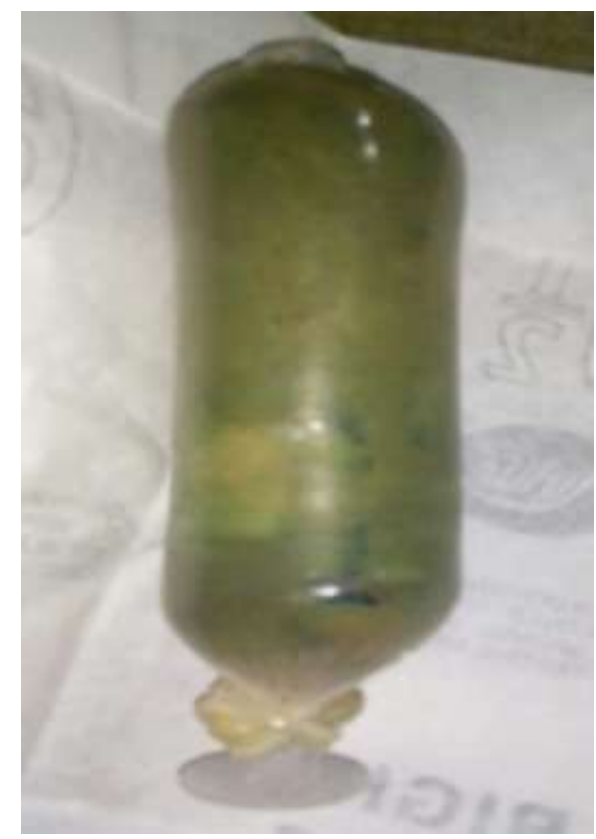

Figure 9: Vaginal mould.

Following graft harvesting and neovagina formation, a stent is needed to apply the graft to the vaginal walls and hold it in place. Both soft and rigid forms have been used. Rigid mould materials have included balsa wood, pyrex, plastic, and synthetic silicone-based materials. Rigid stents may lead to graft loss, fibrosis, contracture, and pressure- related bladder or rectal fistulas. Use of soft stents has decreased the number of these complications.

We use condoms filled with foam rubber as vaginal mould. The size corresponding to the neovagina is selected. Subsequently a condom is applied which is tied on to its open end (Figure 7). Alternatively, $20 \mathrm{ml}$ syringe covered with foam can be used.

The vaginal mould is now covered with amnion graft. The graft is removed from the saline bath. The long axis of the graft is laid parallel to the long axis of the mould. The graft is then draped up and over the mould tip. The lateral edges of the amnion graft are then approximated on either side of the mould using interrupted stitches of 30 catgut. Customizing the mould to the size of the created neovaginal canal is essential. If the mould width is too large, pressure necrosis or inadequate drainage may result, which maylead to tissue necrosis. Once appropriately sized and constructed, the mould is then inserted.

The edges of the labia are then sutured together along the midline using interrupted 2-0 silk sutures to help hold the mould in place for the first 7 postoperative days. A sterile compression dressing is placed on the perineum (Figure 8).

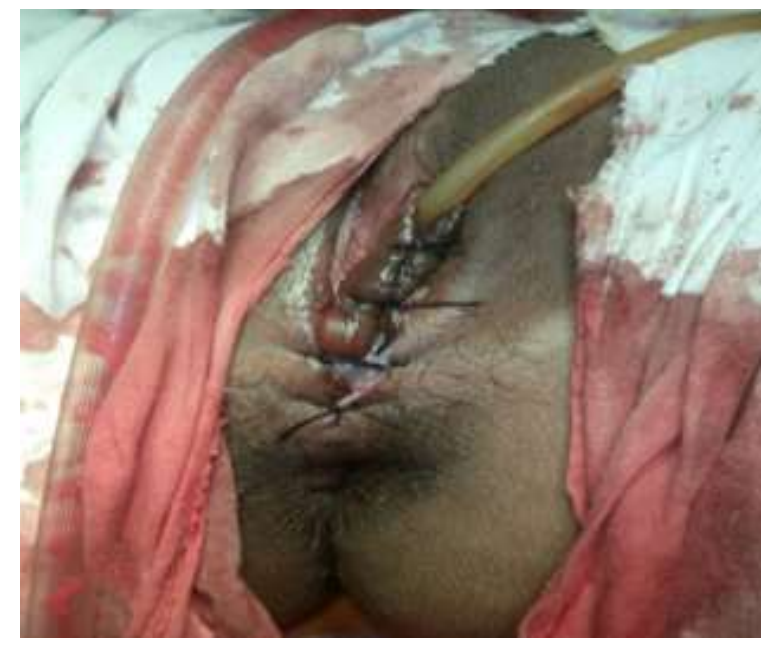

Figure 10: Labial stitches.

\section{Postoperative}

The soft stent and Foley catheter are left in place for 7 days following surgery. To minimize dislodgement of the mould and wound contamination, parenteral antibiotics, a low residue diet (to limit defecation) and daily application of gentamicin drops over labial stitches was done.

Mould removal was done after 7 days in operating room in dorsal lithotomy position. Stitches in the labia are cut, and the mould is removed. To lessen the risk of graft 
avulsion, irrigation is used to reduce adherence between graft and mould. The new cavity formed is carefully washed with warm saline and inspected for graft uptake, any raw area or formation of granulation tissue (Figures 13 and 14).

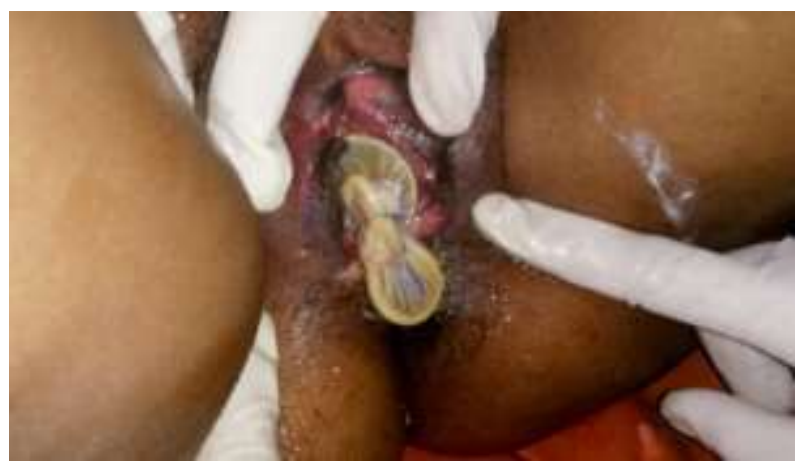

Figure 11: Removal of vaginal mould after 7 days.

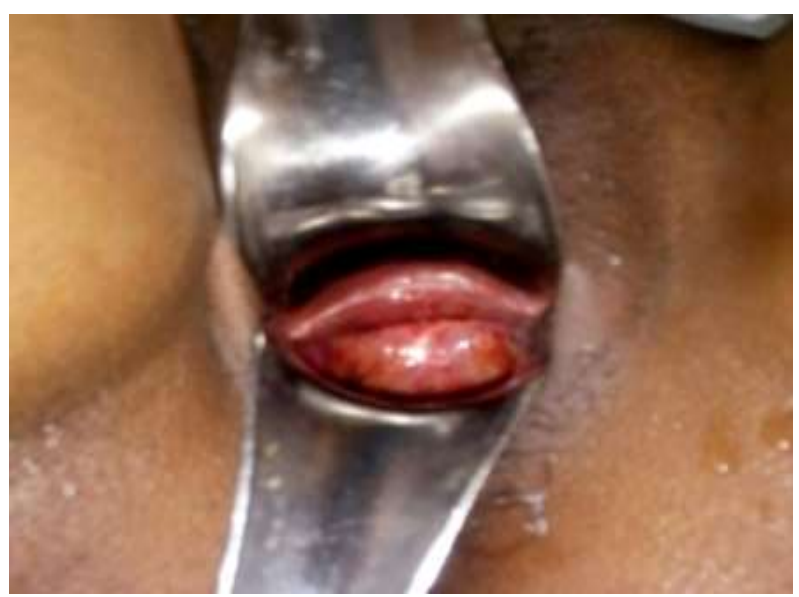

Figure 12: Amnion lined neovagina.

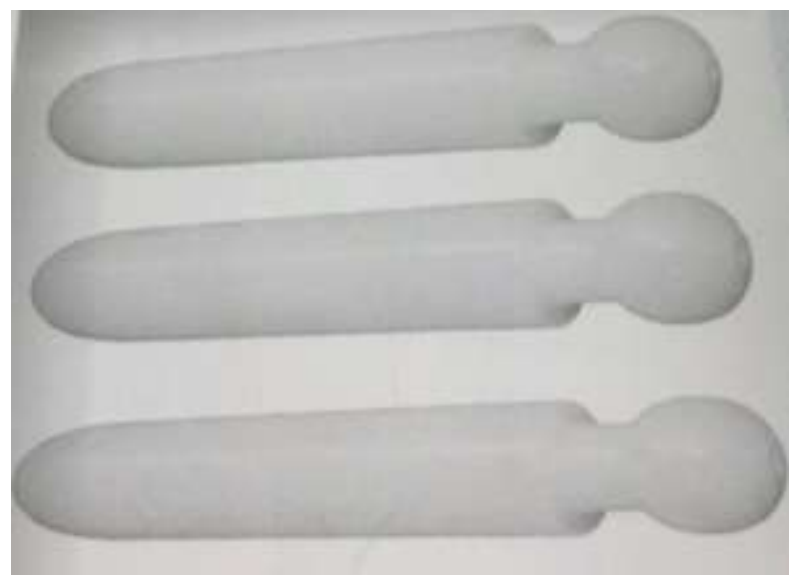

Figure 13: Stanley vaginal dilator set (tufts).

\section{Follow up}

For the first six weeks following surgery, the dilator is worn continuously except during defecation. During the subsequent 6 weeks, it is used only at night. Following these initial 3 months, patients are then instructed to either wear the dilator at night or engage in intercourse twice each week (Figure 12).

\section{Amnion}

The human amniotic membrane is a readily available, cost-free, protective biological dressing for wounds and burns. It has also been shown to stimulate granulation tissue and new vessel formation in chronic venous leg ulcers by producing angiogenic factors. The amnion does not express histocompatibility antigens and there is no evidence of immune rejection when amnion is implanted subcutaneously. These features make the amnion a perfect tissue graft for covering the artificially dissected vagina. Amnion also produces lysozyme which is bactericidal. When an uncontaminated amniotic mesenchymal surface is applied to the raw surface of the dissected vaginal tunnel, it adheres firmly. Protects the underlying granulation tissue and facilitates epithelialization. The human amniotic epithelium is capable of complete metaplasia into mature squamous epithelium, but the mechanism of this cellular transformation is unknown. ${ }^{7,10}$

\section{RESULTS}

Of all the patients operated by us (8) all the patients had wonderful graft uptake. There was a unique complication in one patient which needs to be mentioned here. On the 8 postop days labial stitches were removed and the whole neovagina was visualized. The graft was well lining the cavity and there was no unhealthy discharge. A fresh mould was inserted and patient was shifted back to ward. After 4-6 hours when the patient failed to pass urine, she was recatheterized by the junior resident. She felt a resistance just beyond the external urethral meatus, and using more force she inserted the catheter. Clear urine was drained.

The patient was reexamined the next day in OT, after removing the mould. A small 5-6 mm rent was seen in the anterior vaginal wall through which Foleys catheter was felt. Anatomically, it appeared as urethrovaginal fistula but to confirm methylene blue dye test was done and vesicovaginal fistula was ruled out. Urologist opinion was taken who advised continuous catheterization for 3 months followed by fistula repair, if present. A primary repair at that time would fail as the neovaginal walls do not have enough strength for good healing.

In spite of this as it was a small rent, two stitches using 30 vicryl were applied and a smaller mould was inserted to keep the neovagina patent. Continuous cathterisation for 14 days was done. Daily mould change was done. The fistula healed beautifully and after two weeks catheter was removed. The patient had no urinary complaints in subsequent visits. ${ }^{4,9,11}$ 


\section{DISCUSSION}

A new vagina can be formed either by intermittent vaginal dilatations (non-surgical methods) or by vaginoplasty (surgical method). The non-surgical method may be useful in certain cases of primary vaginal atresia. However, majority of patients require vaginal reconstruction. The cooperation of patient is of paramount importance in vaginoplasty. The most important step is to maintain the vaginal space during contraction period of wound healing and this is accomplished by effective moulds/stents.

The major indications of vaginal reconstruction are congenital absence of vagina (Rokitansky-Kuster-HauserMayer (RKHM) syndrome, gynaecological tumour excision, male to female sex surgery and secondary vaginal atresia.

Since centuries, surgical creation of an artificial vagina is being attempted with varying success. Columbus was perhaps the first to describe the congenital absence of vagina in 1559. Dupuytrens was the first to report vaginoplasty using tampons to maintain patency. Abbes were the first to cover the mould with split thickness skin graft. However, it was McIndoe and Bannister who emphasized the need for dissecting adequqte space, inlaying split thickness skin grafts over the molds and most importantly continuous and prolonged dilatation during the contractile phase of healing. Other surgical methods include constructing neovaginas out of sigmoid colon, ileum, caecum, pudental thigh flaps, fasciocutaneous flaps, gracilis myocutaneous flaps, labia minora flaps, flaps raised following tissue expansion of the labial pocket, peritoneum and bladder mucosa, amnion, the interceed absorbable adhesion barrier, autologus buccal mucosa and lately artificial dermis and recombinant basic fibroblast growth factor have been used. Free skin grafts, peritoneal grafts, local skin flaps and bladder mucosal grafts may scar the patients. Bowel segments have the disadvantages of abdominal scarring and the possibility of bowel obstruction, secretions, unpleasant odor and mucosal ulceration.

\section{CONCLUSION}

Lack of mullerian development (MRKH syndrome) is a common cause of primary amenorrhea, resumption of menstruation and fertility potential is not possible.
Creation of vagina can be done for sexual function. Vagina can be created either nonsurgically by progressive dilatation (Frank's method) or surgically, a primary corrective surgery however has great benefits.

\section{Funding: No funding sources}

Conflict of interest: None declared

Ethical approval: The study was approved by the Institutional Ethics Committee

\section{REFERENCES}

1. Parikh MN. Congenital absence of vagina: MRKH syndrome. Obstet Gynecol India. 2000;501:128-38.

2. Simman R, Jackson I, Andrus L. Refabricated buccal mucosa-lined flap in an animal model that could be used for vaginal reconstruction. Plast Reconstr Surg. 2002;109:1044-9.

3. Ruttgers H, Leucht W, Kubli F. Secondary vaginal atresia: a case report Am J Obstet Gynecol. 1982;143:595-6.

4. Stumpf PG. Stenosis and fistulae with neglected vaginal foreign bodies: a case report. J Reprod Med. 1985;30:559-60.

5. Lin WC, Chang CYY, Shen YY, Tsai HD. Use of autologus buccal mucosa for vaginoplasty: a study of eight cases. Hum Reprod. 2003;18:604-7.

6. Hensle TW, Reiley EA. Vaginal replacement in children and young adults. J Urol. 1998;159:1035-8.

7. Nisolle M, Donnez J. Vaginoplasty using amniotic membranes in cases of vaginal agenesis or after vaginectomy. J Gynecol Surg. 1992;8:25-30.

8. Williams's gynecology. Second edition, Hoffman, Schorge, Schaffer. McGraw-Hill. 2012.

9. Salhan S. Text book of Gynaecology, First edition, 2011, Jaypee publication.

10. Nagrath. Single surgical procedures in obstetrics and gynaecology-32: Congenital anomalies; Jaypee publication. 2013-08.

11. Sharma D, Dahiya K, Chechi K, Sirohiwal D. Journal of gynaecologic surgery. 2008;24(2):61-6.

Cite this article as: Chaudhary R, Dhama V, Singh S, Azad R. Vaginoplasty in mayer Rokitansky-

Kuster-Hauser syndrome using amnion: a case series. Int J Reprod Contracept Obstet Gynecol 2016;5:3832-9. 